\title{
Retraction Note: Efficacy of vitamin C for the prevention and treatment of upper respiratory tract infection. A meta-analysis in children
}

\author{
Philippe Vorilhon ${ }^{1,2}(\mathbb{0})$ Bastien Arpajou ${ }^{1} \cdot$ Hélène Vaillant Roussel ${ }^{1,2} \cdot$ Étienne Merlin $^{3,4} \cdot$ Bruno Pereira $^{5}$. \\ Aurélie Cabaillot $^{1}$
}

Published online: 14 May 2021

(c) Springer-Verlag GmbH Germany, part of Springer Nature 2021

\section{Retraction Note to: \\ European Journal of Clinical Pharmacology (2019) 75:303-311 \\ https://doi.org/10.1007/s00228-018-2601-7}

The Editor-in-chief and the authors retract the following article [1].

After publication, a reader has identified some mistakes in the data reported, these include the following:

(i) Inclusion of studies which did not met strict inclusion criteria: one with pseudo randomization allocation [2] and another one with the intervention group receiving vitamin $\mathrm{C}$ and herbal preparation containing Echinacea and propolis [3] which is relatively heavily weighted and has one of the strongest effects in the analysis of primary and secondary outcomes.

The original article can be found online at https://doi.org/10.1007/ s00228-018-2601-7.

Philippe Vorilhon

philippe.vorilhon@uca.fr

1 Department of General Practice, Clermont Auvergne University, Faculty of Medicine, 28 Place Henri Dunant, 63000 Clermont-Ferrand, France

2 UPU ACCePPT, Clermont Auvergne University, 28 Place Henri Dunant, 63000 Clermont-Ferrand, France

3 Pediatrics, Clermont-Ferrand University Hospital, Clermont-Ferrand, France

4 Pediatrics, INSERM-CIC 1405, Clermont-Ferrand University Hospital, 1 Place Lucie \& Raymond Aubrac, 63003 Clermont-Ferrand Cedex 1, France

5 Biostatistics Unit (Clinical Research and Innovation Department), Clermont-Ferrand University Hospital, 58 Rue Montalembert, 63000 Clermont-Ferrand, France (ii) Some data, numbers of upper respiratory tract infection (URTI) episodes and standard-deviations of URTI durations, extracted from studies were erroneous.

(iii) Some statistical approaches performed in our metaanalysis are not appropriate and have not been described accurately. These errors are likely to invalidate the results.

The authors acknowledge and apologize for these involuntary errors. For these reasons, the article has been retracted.

\section{References}

1. Vorilhon P, Arpajou B, Vaillant Roussel H, Merlin É, Pereira B, Cabaillot A (2019) Efficacy of vitamin C for the prevention and treatment of upper respiratory tract infection. A meta-analysis in children. Eur J Clin Pharmacol 75(3):303-311

2. Coulehan JL, Reisinger KS, Rogers KD, Bradley DW (1974) Vitamin $\mathrm{C}$ prophylaxis in a boarding school. N Engl J Med 290(1):6-10

3. Cohen HA, Varsano I, Kahan E, Sarrell EM, Uziel Y (2004) Effectiveness of an herbal preparation containing echinacea, propolis, and vitamin $\mathrm{C}$ in preventing respiratory tract infections in children: a randomized, double-blind, placebo-controlled, multicenter study. Arch Pediatr Adolesc Med 158(3):217-221 